\title{
Intraoperative occlusion of left coronary ostium after aortic repair detected by transesophageal echocardiography
}

\author{
Kazumasa Orihashi, MD, Taiichi Takasaki, MD, Masamichi Ozawa, MD, and Taijiro Sueda, MD, \\ Hiroshima, Japan
}

In the surgical treatment of acute aortic dissection, new onset of malperfusion in one or more of the vital organs is not uncommon. Such events are often unpredictable, but they necessitate immediate decision making. ${ }^{1,2}$ We report a case involving sudden occlusion of the left coronary artery ostia diagnosed with intraoperative transesophageal echocardiography (TEE) after uneventful weaning from cardiopulmonary bypass. We also discuss intraoperative strategies.

\section{CLINICAL SUMMARY}

A 60-year-old male patient underwent aortic repair for acute type A dissection complicated with pericardial tamponade. TEE after induction of anesthesia showed the following: (1) aortic dissection in the ascending to abdominal aorta, (2) left ventricular hypertrophy without asynergy, (3) pericardial fluid, (4) competent aortic valve, and (5)

From the Department of Cardiovascular Surgery, Hiroshima University Hospital, Hiroshima, Japan.

Disclosures: Authors have nothing to disclose with regard to commercial support.

Received for publication May 26, 2011; revisions received Aug 4, 2011; accepted for publication Aug 24, 2011; available ahead of print Sept 26, 2011.

Address for reprints: Kazumasa Orihashi, MD, Department of Surgery II, Kōchi Medical School, Kohasu, Oko-cho, Nankoku-city, Kōchi 783-8505, Japan (E-mail: orihashik@kochi-u.ac.jp).

J Thorac Cardiovasc Surg 2011;142:e205-6 $0022-5223 / \$ 36.00$

Copyright (c) 2011 by The American Association for Thoracic Surgery doi:10.1016/j.jtcvs.2011.08.022 intact coronary arteries and sinus of Valsalva (Figure 1, $A$ and $B)$. Under circulatory arrest $\left(25^{\circ} \mathrm{C}\right)$ and selective cerebral perfusion, the ascending aorta was replaced with a $22-\mathrm{mm}$ vascular graft after the aortic stump was reinforced with a pair of felt strips. The TEE assessment after weaning from bypass showed no unusual findings (Figure 1, C).

Before chest closure, however, systemic blood pressure suddenly dropped to $30 \mathrm{~mm} \mathrm{Hg}$. TEE revealed akinesis in the anterior to posterior left ventricular wall. Blood flow was undetectable in the left coronary artery, and localized dissection was found in the noncoronary and left coronary sinuses of Valsalva (Figure 1,D). We connected the patient to cardiopulmonary bypass and anastomosed a saphenous vein graft to the left anterior descending artery for selective perfusion. Because retrograde dissection from the proximal anastomosis of the aorta was likely responsible, we decided to repair the sinus of Valsalva. On aortotomy, the wall of the sinus of Valsalva was seen to be bulging, but the left coronary ostium was patent without any intimal tearing, and the cardioplegic solution could be infused without resistance. A pair of pledgeted 4-0 polypropylene sutures were placed at the noncoronary and left coronary sinuses of Valsalva (Figure 2, A).

The patient was weaned from bypass without difficulty. TEE demonstrated the following: (1) detectable blood flow in the left coronary artery, (2) restored ventricular contraction without asynergy, (3) no aortic regurgitation, and

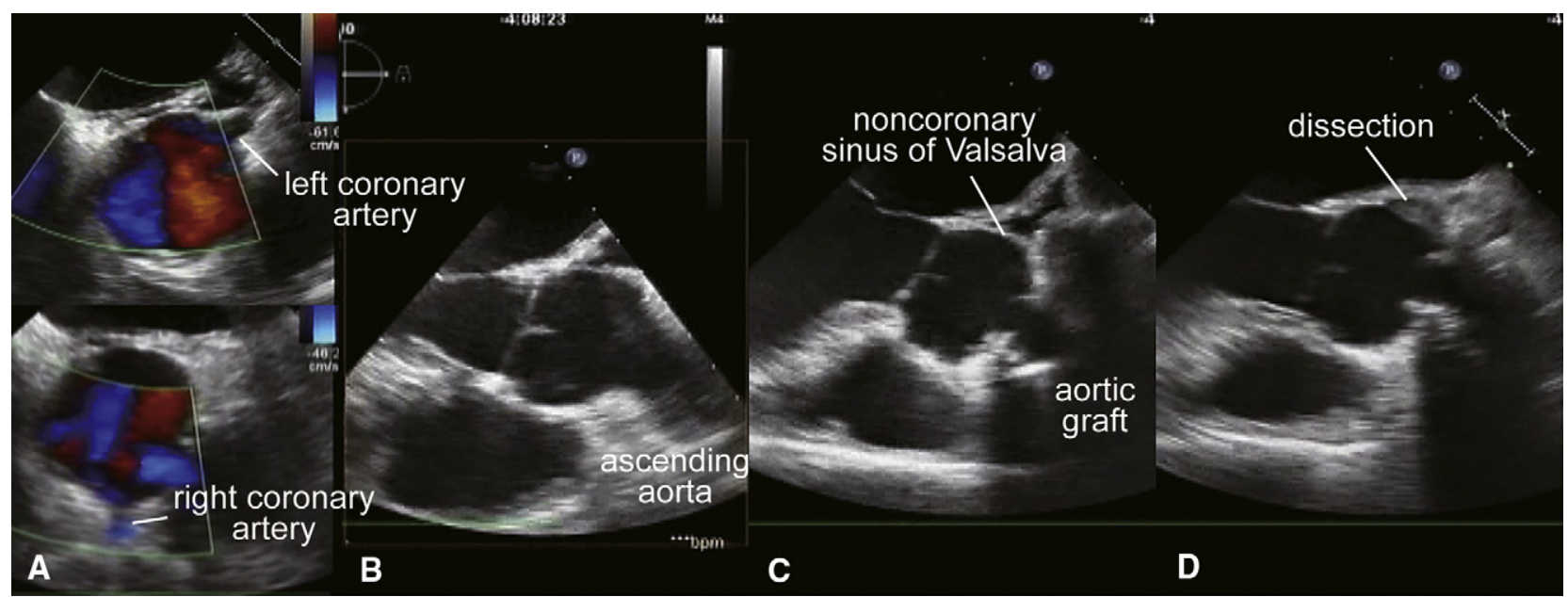

FIGURE 1. Transesophageal echocardiograms showing intact coronary arteries and sinus of Valsalva (A, B) and after aortic repair (C). D, New dissection in the noncoronary sinus of Valsalva when the patient was hypotensive. 

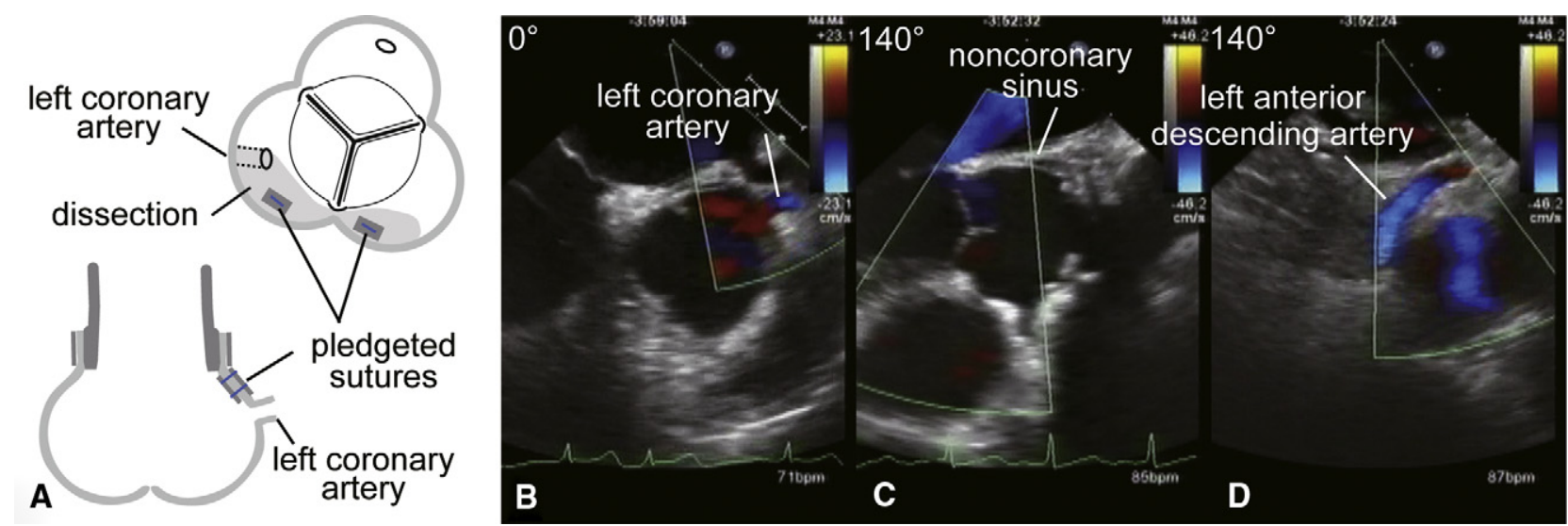

FIGURE 2. Illustration of repair on the sinus of Valsalva (A) and transesophageal echocardiograms showing aortic root (B, C) and blood flow in the left anterior descending artery after repair (D).

(4) flattened false lumen in the wall of the sinus of Valsalva (Figure 2, B-D). Postoperative course was uneventful, and computed tomography showed patent coronary ostium and saphenous vein graft.

\section{DISCUSSION}

This event was probably caused by new dissection that originated from the aortic anastomosis site, extended retrogradely, and collapsed the lumen of the coronary ostium. While such an event is unpredictable, it necessitates immediate diagnosis and appropriate treatment without delay. In such a situation, TEE can be helpful for making a diagnosis by assessing the coronary arteries, including the left anterior descending and left circumflex arteries. ${ }^{3}$

Impaired left ventricular contraction indicated malperfusion in the left coronary artery, and we immediately revascularized the left anterior descending coronary artery and selectively perfused it to minimize the ischemic time. Coronary revascularization did not solve the problem in the sinus of Valsalva, however, and a risk of extension of dissection to the right coronary artery and/or aortic annulus remained. We therefore decided to add a repair on the sinus of Valsalva. Subsequent TEE assessment confirmed the adequacy of the measures taken.

Although such unpredictable events can occur, the current case demonstrates the utility of intraoperative diagnostic imaging, which facilitates appropriate decision making as well as subsequent evaluation of the echocardiographically based strategy.

\section{References}

1. Orihashi K, Sueda T, Okada K, Takahashi S. Compressed true lumen in the innominate artery: a pitfall of right axillary arterial perfusion in acute aortic dissection. $J$ Thorac Cardiovasc Surg. 2009;137:242-3.

2. Orihashi K, Sueda T, Okada K, Imai K. Detection and monitoring of complications associated with femoral or axillary arterial cannulation for surgical repair of aortic dissection. J Cardiothorac Vasc Anesth. 2006;20:20-5.

3. Ender J, Selbach M, Borger MA, Krohmer E, Falk V, Kaisers UX, et al. Echocardiographic identification of iatrogenic injury of the circumflex artery during minimally invasive mitral valve repair. Ann Thorac Surg. 2010;89:1866-72. 